\title{
Droplet formation in a T-shaped microfluidic junction
}

\author{
Haihu Liu and Yonghao Zhang* \\ Department of Mechanical Engineering, \\ University of Strathclyde, Glasgow G1 1 XJ, UK
}

(Dated: April 27, 2009)

\begin{abstract}
Using a phase-field model to describe fluid/fluid inter-facial dynamics and a lattice Boltzmann model to address hydrodynamics, 2 dimensional (2D) numerical simulations have been performed to understand the mechanisms of droplet formation in microfluidic T-junction. Although $2 D$ simulations may not capture underlying physics quantitatively, our findings will help to clarify controversial experimental observations and identify new physical mechanisms. We have systematically examined the influence of capillary number, flow rate ratio, viscosity ratio and contact angle in the droplet generation process. We clearly observe that the transition from the squeezing regime to the dripping regime occurs at a critical capillary number of 0.018, which is independent of flow rate ratio, viscosity ratio and contact angle. In the squeezing regime, the squeezing pressure plays a dominant role in the droplet breakup process, which arises when the emerging interface obstructs the main channel. The droplet size depends on both the capillary number and the flow rate ratio, but is independent of the viscosity ratio under completely hydrophobic wetting conditions. In the dripping regime, the droplet size will be significantly influenced by the viscosity ratio as well as the built-up squeezing pressure. When the capillary number increases, the droplet size becomes less dependent on the flow rate ratio. The contact angle also affects the droplet shape, size and detachment point, especially at small capillary numbers. More hydrophobic wetting properties are expected to produce smaller droplets. Interestingly, the droplet size is dependent on the viscosity ratio only for less hydrophobic wetting conditions.
\end{abstract}

PACS numbers: 47.11-j, 47.55.db, 47.55.df

*Electronic address: yonghao.zhang@strath.ac.uk 


\section{INTRODUCTION}

Rapid development of micro-fabrication techniques has facilitated a broad range of microfluidic applications in biological and chemical research and associated industries [1-3]. Microdroplet technology has recently emerged as a promising new paradigm for biological and chemical analysis, with each droplet acting as an individual chemical reactor. Sample encapsulation in the form of droplets avoids the sample dilution caused by Taylor dispersion [4], and increases mixing performance [5]. In addition, it can avoid sample/surface interaction thus eliminate surface adsorption and cross sample contamination. Although many droplet-based microfluidic devices have been developed, users have not yet embraced this technology. One major reason is that dynamics of droplet in microchennels has not well understood, which hinders device optimization and operation. Here, we aim to address this challenge and focus on understanding the dynamics process of droplet generation in a T-junction.

T-junctions have been commonly used to generate droplets in microfluidic devices e.g. [514]. The channel geometries have been found to play an important role in the droplet formation process. For example, Garstecki et al. [8] identified a squeezing mechanism due to confined geometry in droplet formation process, which does not exist in an unbounded flow condition. However, the currently available experimental data are sporadic. Various materials are used to fabricate the channels with different dimensions, while the experiments are operated under a wide range of flow conditions with different fluids. Consequently, the information is fragmented, which leads to inconclusive and even contradictory findings. Many coupled factors will affect the droplet formation process, e.g. the inter-facial tension, the wetting properties of solid surface, the volumetric flow rates of both immiscible fluids and their viscosities, so that the droplet dynamics in microdevices is very complicated. While experimental work has helped to understand underlying physics, experiments at such small scale are still difficult. For example, it is challenging to accurately measure droplet size, pressure and velocity fields, and droplet deformation, breakup and coalescence. Since numerical simulation can provide more detailed information on droplet dynamics, computational approach has emerged to investigate droplet generation process, e.g. [15-18]. Rich information can be extracted from simulation data, e.g. De Menech et al. [17] identified three distinct mechanisms i.e. squeezing, dripping and jetting. However, there is currently no simula- 
tion method available that captures both intermolecular and hydrodynamic effects in droplet dynamics, within the reach of current computational technology. It is still challenging to numerically simulate droplet generation, transportation, and interactions with other droplets and surface. While the front tracking methods are not suitable for droplet breakup and coalescence, the interface capturing methods such as volume-of-fluid and level set methods will experience numerical instability at the interface regime when the inter-facial tension becomes a dominant factor in microdroplet behavior [19]. Recently developed lattice Boltzmann (LB) method is known to be able to model inter-facial interactions while incorporates fluid flow as a system feature [20]. It is a pseudo-molecular method tracking evolutions of the distribution function of an assembly of molecules and built upon microscopic models and mesoscopic kinetic equations [21]. Its mesoscopic nature can provide many advantages of molecular dynamics, making the LB method especially useful for simulating droplet dynamics. Here, an improved multiphase LB model, using the phase field theory to describe inter-facial interactions, will be employed to investigate the droplet formation in a microfluidic T-junction. The influence of capillary number, flow rate ratio, viscosity ratio and contact angle on droplet breakup, size and detachment are to be systematically studied. Similar numerical investigation has recently been performed in 3 dimensions by De Menech et al. [17] where the phase-field model was coupled with the Navier-Stokes equations to resolve inter-facial dynamics as well as hydrodynamics.

\section{NUMERICAL MODEL}

A unifying feature of all the phase-field models is the existence of a free-energy functional, which not only determines the equilibrium properties, but also strongly influences the dynamics of a multiphase system. The transport of physical quantities can be linked to free-energy functional by a generalized hydrodynamic theory [22], and thus the interface can be captured automatically in the simulation. Here, we describe the phase field model for a two-phase system and the lattice Boltzmann model, which are used in our simulation. In particular, we emphasize the implementation of the inlet and outlet boundary conditions and the fluid/surface interactions. 


\section{A. Free energy theory}

We consider a binary incompressible fluid consisting of $A$ and $B$ molecules with the phase orders into an $A$-rich phase and a $B$-rich phase below a critical temperature. The Landau free energy [23] is used to describe the equilibrium properties of the fluid:

$$
F(\phi, \nabla \phi)=\int\left[\Psi(\phi)+\frac{1}{2} \kappa|\nabla \phi|^{2}+\rho c_{s}^{2} \ln \rho\right] d V
$$

where $\rho_{A}, \rho_{B}$ are the number densities of $A$ and $B$, respectively; $\rho=\rho_{A}+\rho_{B}$ is the total density; $\phi=\left(\rho_{B}-\rho_{A}\right) / \rho$ is the the order parameter, which describes the normalized density difference in the two fluids; $\Psi(\phi)$ is the bulk free energy density having a double well form, $\Psi(\phi)=\frac{1}{4} a\left(\phi^{2}-1\right)^{2} ; \frac{1}{2} \kappa|\nabla \phi|^{2}$ is the inter-facial energy density, which is linked to the interfacial tension. The final term in the free energy, which depends only on the density $\rho$, is introduced to enforce incompressibility.

The chemical potential $\mu$ is defined as the variational derivative of the free energy functional with respect to the order parameter, i.e.,

$$
\mu=\delta F / \delta \phi=\Psi^{\prime}(\phi)-\kappa \nabla^{2} \phi=a \phi\left(\phi^{2}-1\right)-\kappa \nabla^{2} \phi
$$

The equilibrium interface profile can be obtained from Eq. (2) at $\mu=0$, which leads to two stable uniform solutions $\phi= \pm 1$ representing the coexisting bulk phases. The onedimensional nonuniform solution, e.g. the $x$-direction along the gradient of $\phi$, is given by

$$
\phi(x)=\tanh (x / \xi)
$$

where $\xi$ is a parameter proportional to the interface thickness, which is defined as

$$
\xi=\sqrt{2 \kappa / a}
$$

Since the inter-facial tension $\sigma$ can be interpreted as the excess free energy per unit interface area for a plane interface in equilibrium [24], it can be obtained as

$$
\sigma=\kappa \int_{-\infty}^{+\infty}\left(\frac{\mathrm{d} \phi}{\mathrm{d} x}\right)^{2} d x .
$$

With Eq. (3) and Eq. (5), we can get

$$
\sigma=\frac{4 \kappa}{3 \xi}
$$

Eqs.(4) and (6) suggest that we can control the inter-facial tension and the interface thickness through the parameters $\kappa$ and $a$. 


\section{B. Lattice Boltzmann model}

Two distribution functions $g_{i}$ and $f_{i}$ are used to describe the evolution of the order parameter $\phi(\vec{x})$ and the flowfield $\vec{u}(\vec{x})$ respectively, and the evolution equations are:

$$
\begin{aligned}
& f_{i}\left(\vec{x}+\vec{e}_{i} \delta_{t}, t+\delta_{t}\right)-f_{i}(\vec{x}, t)=\frac{f_{i}^{e q}(\vec{x}, t)-f_{i}(\vec{x}, t)}{\tau_{f}} \\
& \quad+\left(1-\frac{1}{2 \tau_{f}}\right) \frac{\left(\vec{e}_{i}-\vec{u}\right) \cdot \mu \nabla \phi}{c_{s}^{2}} \Gamma_{i}(\vec{u}) \delta_{t}, \\
& g_{i}\left(\vec{x}+\vec{e}_{i} \delta_{t}, t+\delta_{t}\right)-g_{i}(\vec{x}, t)=\frac{g_{i}^{e q}(\vec{x}, t)-g_{i}(\vec{x}, t)}{\tau_{g}},
\end{aligned}
$$

where

$$
\Gamma_{i}(\vec{u})=w_{i}\left[1+\frac{e_{i \alpha} u_{\alpha}}{c_{s}^{2}}+\frac{u_{\alpha} u_{\beta}\left(e_{i \alpha} e_{i \beta}-c_{s}^{2} \delta_{\alpha \beta}\right)}{2 c_{s}^{4}}\right],
$$

and $c_{s}$ is the lattice speed of sound, which is $c / \sqrt{3}$ for the D2Q9 model [25]; $\delta_{t}$ is the time step and $w_{i}$ are the weight factors associated with the lattice velocity vectors $\vec{e}_{i} ; \tau_{f}$ and $\tau_{g}$ are the relaxation parameters related to the fluid viscosity and the mobility respectively. The equilibrium distribution functions, $f_{i}^{e q}$ and $g_{i}^{e q}$, are given as below,

$$
\begin{aligned}
& f_{i}^{e q}=w_{i}\left[\frac{p}{c_{s}^{2}}+\rho\left(\frac{e_{i \alpha} u_{\alpha}}{c_{s}^{2}}+\frac{u_{\alpha} u_{\beta}\left(e_{i \alpha} e_{i \beta}-c_{s}^{2} \delta_{\alpha \beta}\right)}{2 c_{s}^{4}}\right)\right], \\
& g_{i}^{e q}=w_{i}\left[A_{i}+\phi\left(\frac{e_{i \alpha} u_{\alpha}}{c_{s}^{2}}+\frac{u_{\alpha} u_{\beta}\left(e_{i \alpha} e_{i \beta}-c_{s}^{2} \delta_{\alpha \beta}\right)}{2 c_{s}^{4}}\right)\right],
\end{aligned}
$$

where the coefficients $A_{i}$ are given by

$$
A_{i}= \begin{cases}\Gamma \mu / c_{s}^{2} & (i>0) \\ {\left[\phi-\left(1-w_{0}\right) \Gamma \mu / c_{s}^{2}\right] / w_{0}} & (i=0),\end{cases}
$$

where $\Gamma$ is a parameter related to the Cahn-Hilliard mobility $M$, i.e. $M=\Gamma\left(\tau_{g}-1 / 2\right) \delta_{t}$.

In the following simulations, the densities of the phases $A$ and $B$ are assumed to be equal because the density difference in the commonly-used water droplet in oil is small. We set $\tau_{g}=1 /(3-\sqrt{3})$ to minimize numerical errors of the convection-diffusion scheme [26]. To account for unequal viscosities of the two phases, we define the viscosity $\eta$ as a linear function of the order parameter:

$$
\eta(\phi)=\frac{1-\phi}{2} \eta_{A}+\frac{1+\phi}{2} \eta_{B}
$$

In this way, $\eta$ automatically changes across the interface with a profile similar to the tanh function. In Eq. (13), $\eta_{A}$ and $\eta_{B}$ are the viscosities of the phase $A$ and $B$. The local 
relaxation time $\tau_{f}$ can be calculated from the local viscosity by:

$$
\eta(\phi)=\rho c_{s}^{2}\left[\tau_{f}(\phi)-1 / 2\right] \delta_{t}
$$

After the propagation step, the macroscopic variables can be calculated as:

$$
\begin{aligned}
\phi & =\sum_{i} g_{i}, \\
\frac{p}{c_{s}^{2}} & =\sum_{i} f_{i}, \\
\rho \vec{u} & =\sum_{i} f_{i} \vec{e}_{i}+\frac{1}{2} \mu \nabla \phi .
\end{aligned}
$$

Using the Chapman-Enskog multiscale expansion, the Navier-Stokes equations and the Cahn-Hilliard equation can be obtained:

$$
\begin{gathered}
\nabla \cdot \vec{u}=0, \\
\rho\left(\partial_{t} \vec{u}+\vec{u} \cdot \nabla \vec{u}\right)=-\nabla p+\nabla \cdot\left[\eta\left(\nabla \vec{u}+\nabla \vec{u}^{\mathrm{T}}\right)\right]+\mu \nabla \phi, \\
\partial_{t} \phi+\vec{u} \cdot \nabla \phi=\nabla \cdot(M \nabla \mu) .
\end{gathered}
$$

Note that the Navier-Stokes equations converge to the classical sharp interface behavior as the interface thickness reduces towards zero along with the diffusivity $1 / \mathrm{Pe}$, where $\mathrm{Pe}$ is the Peclet number defined as:

$$
\mathrm{Pe}=U L / M a,
$$

where $U$ and $L$ are the characteristic velocity and length of the system.

In the numerical solution, a sufficient number of grids are required to avoid spurious currents and numerical instability at the interface. For a finite interface thickness, straining flows can thicken or thin the interface, which must be balanced by diffusion. Meanwhile, large diffusion will excessively damp the flow. Therefore, Peclet number, which indicates the diffusivity, must be appropriately chosen [15]. In our simulations, we compromise to have $\xi$ of two lattice grids, so that the interface is resolved typically $5 \sim 6$ grids, and $\mathrm{Pe}$ is of $O(10) \sim O(100)$.

\section{Boundary conditions}

No-slip boundary condition is applied at solid walls using half-way bounceback [20], which can prevent the boundary "mass leakage", especially for a flow with small velocity. If the 
fluid node $\vec{x}$ is a boundary node, its links are divided into two groups: boundary links (BL) and fluid links (FL) [27]. With a given inlet volumetric flow rate, $Q_{i n}$, we impose the following inlet boundary condition:

$$
f_{i}\left(\vec{x}, t+\delta_{t}\right)= \begin{cases}f_{i^{*}}\left(\vec{x}, t^{+}\right)+2 w_{i} \rho Q_{i n} / c_{s}^{2} & \text { if } i^{*} \text { is BL, } \\ f_{i}\left(\vec{x}+\vec{e}_{i^{*}}, t^{+}\right) & \text {otherwise }\end{cases}
$$

where $\vec{e}_{i^{*}}=-\vec{e}_{i}, t^{+}$is the time immediately after the collision, i.e. $t^{+}-t \ll 1$. We adopt the outlet pressure boundary condition developed by Zou and He [28].

The wetting properties are important for fluid/surface interactions. Iwahara et al. [29] proposed an elegant way to implement the wetting boundary condition in a vapor-liquid system. Recently, van der Graaf et al. [16] used the same wetting boundary treatment for a liquid-liquid system. They assume that the wall is a mixture of two fluids, thus having a certain value of the order parameter $\phi_{w}$, so the derivatives of the order parameter at the surface boundary can be calculated using (9 points) regular finite difference stencils [30]. Consequently, the chemical potential and the external force term in Eq. (7) become dependent on the properties of the neighboring solid lattice sites, resulting in a special case of the Cahn boundary condition [31]. Similarly, we use the formula below to assign the order parameter $\phi_{w}$ to the solid lattice site next to the wall:

$$
\cos \left(\theta_{w}\right)=\frac{1}{2} \phi_{w}\left(3-\phi_{w}^{2}\right)
$$

where $\theta_{w}$ is the desired static contact angle, which is related to the inter-facial tensions by the Young's equation:

$$
\cos \left(\theta_{w}\right)=\frac{\sigma_{A w}-\sigma_{B w}}{\sigma},
$$

where $\sigma_{A w}$ or $\sigma_{B w}$ is the inter-facial tension between the fluid phase ( $A$ or $B$ ) and the solid surface.

\section{DROPLET FORMATION IN A T-JUNCTION}

We study droplet formation in a T-junction microchannel, as illustrated in Fig. 1, consisting of a main channel with width $w_{c}$ and a lateral channel with width $w_{d}$. Both $w_{c}$ and $w_{d}$ are $100 \mu \mathrm{m}$ in the current simulations. The continuous phase oil (phase $A$ ) is introduced at the inlet of the main channel, and the dispersed phase water (phase $B$ ) is injected into 


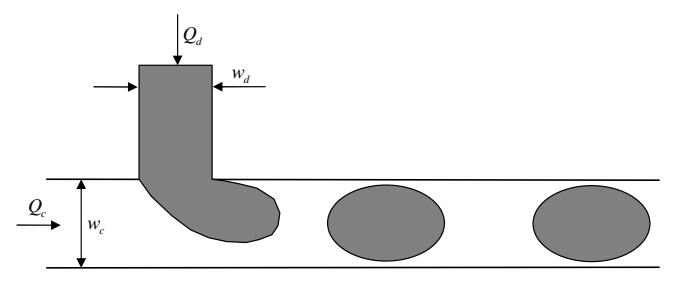

FIG. 1: Schematic illustration of the droplet generation in a microfluidic T-junction, where $w_{c}, w_{d}$ are the widths of the main and lateral channels, and $Q_{c}$ and $Q_{d}$ are the inlet volumetric flow rates of the continuous and dispersed phases.

the lateral channel. Since the phase field calculation will resolve the interface structure, it is computationally too costly for a 3 dimensional (3D) simulation to resolve a typical 1 $\mathrm{nm}$ oil-water interface of a microdroplet. Therefore, 3D phase field calculation will have to artificially enlarge the interface thickness to simulate droplet motion. Since the grid number may significantly affect the simulation results of the phase field method, it is important to minimize the numerical error introduced by the grid number. While the essential physics can still be retained, we run simulations in $2 \mathrm{D}$ with the computational domain consisting of $300 \times 60$ lattices. In all the cases, we find that mesh refinement will lead to results variations no more than $5 \%$. We will now use the subscripts ' $\mathrm{c}$ ' and 'd' to refer to the continuous and dispersed phases respectively.

Flow behavior in a microfluidic T-junction can be classified by a group of dimensionless parameters, which are commonly defined by the experimentally measurable variables e.g. the inter-facial tension, the inlet volumetric flow rates $\left(Q_{c}\right.$ and $\left.Q_{d}\right)$ and viscosities $\left(\eta_{c}\right.$ and $\eta_{d}$ ) of the two fluids. For typical microfluidic flow, the Reynolds number is so small that the inertial effect can be neglected. The Bond number is also negligibly small due to the small density difference of the two immiscible liquids. In contrast, the capillary number (Ca), which describes relative importance of the viscosity and the inter-facial tension, is a most important parameter to understand droplet generation. Here it is defined by the average inlet velocity $u_{c}$ and the viscosity $\eta_{c}$ of the continuous phase, and the inter-facial tension $\sigma$ as

$$
\mathrm{Ca}=\frac{\eta_{c} u_{c}}{\sigma}=\frac{\eta_{c} Q_{c}}{\sigma w_{c}}
$$

Another important dimensionless parameter is the ratio of flow rates $\left(Q=Q_{d} / Q_{c}\right)$. In addition, the viscosity ratio $\left(\lambda=\eta_{d} / \eta_{c}\right)$ and fluid/surface interaction have been experimentally 


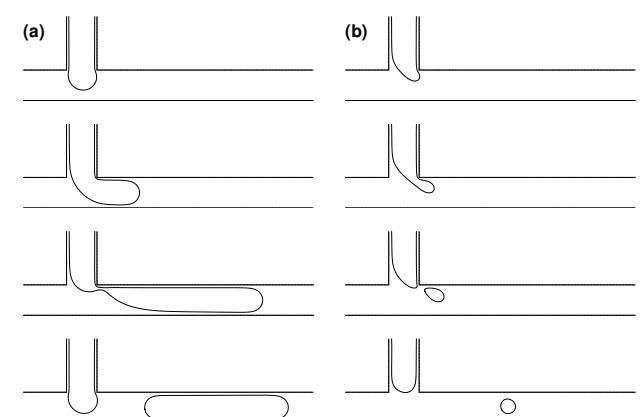

FIG. 2: The snapshots of droplet formation: (a) $\mathrm{Ca}=0.00056, Q_{d}=0.004$; and (b) $\mathrm{Ca}=0.059$, $Q_{d}=0.008$.

observed to play important roles in droplet formation process [32, 33]. In order to achieve consistent droplet breakup, it is necessary that the continuous phase liquid should preferentially wet the walls. The magnitude of wettability will be described by a static contact angle $\theta_{w}$. Here, we will examine the roles of these parameters in droplet formation.

\section{A. Influence of capillary number}

A series of simulations are performed with the inter-facial tension $\sigma=0.0036$, the viscosity of the continuous phase $\eta_{c}=0.016$ and the viscosity ratio $\lambda=1 / 4$. The corresponding dimensional inter-facial tension and viscosity of the continuous phase are $0.01125 \mathrm{Nm}^{-1}$ and 0.02 Pas respectively. The densities of both fluids are assumed to be unity. The contact angle $\theta_{w}=180^{\circ}$ so that the continuous phase fluid completely wets the walls, while the dispersed phase fluid is non-wetting. In order to compare our simulation results with the experimental data $[11,12,34]$, we choose $Q_{d}=0.004,0.006$ and 0.008 in the simulations. For convenience, all quantities are expressed in the lattice units except the droplet diameter, which is in the physical unit.

Fig. 2 shows the snapshots of droplet formation in the T-junction. The droplet deforms before detachment, and the necking of the dispersed phase is initiated once the continuous phase fluid intrudes into the upstream of the lateral channel. The intrusion of the continuous phase accentuates the influence of the contact line dynamics, which is thought to be indispensable for the droplet detachment. Fig. 2 shows that the necking occurs soon after the dispersed phase streams into the main channel and the droplets are produced when Ca 


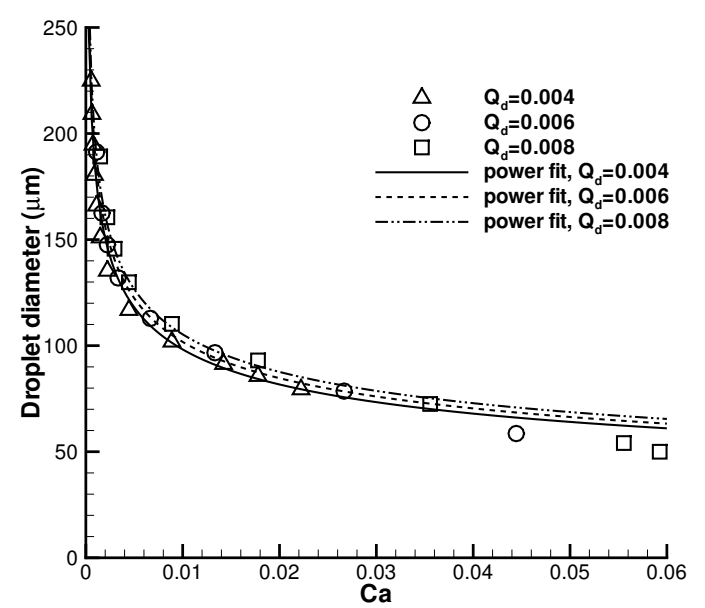

FIG. 3: The effect of capillary number on the droplet diameter at the dispersed phase flow rates $Q_{d}$ of $4 \times 10^{-3}, 6 \times 10^{-3}$ and $8 \times 10^{-3}$ respectively. The lines represent the power fitting of the simulation results

is large (Ca=0.059), while the plugs are formed when Ca is small (Ca=0.00056). This phenomenon has also been observed in the experimental work [11, 12]. Fig. 3 shows that the droplet diameter becomes smaller when Ca increases for a fixed dispersed phase flow rate, and is very weakly dependent on the dispersed phase flow rate. Here, the effective diameter of a non-spherical droplet is calculated as the same as the diameter of a spherical droplet with the same volume.

In both experiment [7] and simulation [16], van der Graaf et al. found that the final droplet volume is a result of a two-stage droplet growth. Initially, the droplet grows to a critical volume $V_{c}$ until the forces exerted on the interface become balanced. Subsequently, the droplet continues to grow for a time $t_{n}$ for necking due to the continuous injection of the dispersed phase fluid. And the final droplet volume $V$ can be described by [7]

$$
V=V_{c}+t_{n} Q_{d}
$$

$V_{c}$ depends only on $\mathrm{Ca}$ and the duration of necking $t_{n}$ and decreases when Ca increases. An empirical relationship was proposed to predict the droplet volume by van der Graaf et al. $[16]$ :

$$
V=V_{c, r e f} C a^{m}+t_{n, r e f} C a^{n} Q_{d}
$$

where $V_{c, r e f}$ and $t_{n, \text { ref }}$ are the reference values at $\mathrm{Ca}=1$ (the droplet detachment process 
is very fast, i.e. $t_{n} \rightarrow 0$ ); the exponents $m$ and $n$ depend on the device geometry. In our simulation, we find that $m=n=-0.78$, while $m=n=-0.75$ were also reported by van der Graaf et al. [16].

\section{B. Influence of flow rate ratio}

Here, we study the influence of flow rate ratio on droplet formation in the T-junction over a broad range of capillary numbers. Three different flow rate ratios $Q=1 / 8,1 / 4$, and $1 / 2$ are used in the simulations. For each flow rate ratio, the capillary number varies from 0.004 to 0.056 , typically found in microfluidic droplet generation. The wetting conditions and the densities of both phases are kept the same as in the previous section. The inter-facial tension $\sigma$ is now set to be 0.005, and the viscosity of the continuous phase fluid $\eta_{c}$ is 0.08 (the corresponding dimensional values are $0.0156 \mathrm{Nm}^{-1}$ and 0.01 Pas respectively). The viscosity ratio $\lambda$ is $1 / 8$ here.

As shown in Fig. 4, when the capillary number is low i.e. $\mathrm{Ca}=0.006$, the incoming dispersed phase fluid tends to occupy the full width of the main channel, and the breakup occurs at the downstream corner of the T-junction. When the capillary number increases i.e. $\mathrm{Ca}=0.032,0.056$, the dispersed phase fluid occupies only part of the main channel, and smaller droplets are formed. According to Ca, we find two distinguished droplet generation regimes i.e. the squeezing and dripping regimes as identified by De Menech et al. [17]. In the squeezing regime when $\mathrm{Ca}$ is small, the pressure buildup in the upstream due to the obstruction of the main channel by the emerging droplet plays a dominant role in "pinching off" the droplet, while the viscous shear force becomes increasingly important in the dripping regime when $\mathrm{Ca}$ increases.

Fig. 4 clearly shows the effect of $Q$ and $\mathrm{Ca}$ on the droplet detachment point. For small $Q$, i.e. $Q=1 / 8$, the droplets are pinched off at the T-junction corner for all the capillary numbers. However, for $Q=1 / 4$ and $1 / 2$, increasing Ca will force the detachment point to move from the corner to the downstream. When $\mathrm{Ca}$ is 0.006 , varying $Q$ from $1 / 8$ to $1 / 2$ does not change the detachment point of the droplet. When $\mathrm{Ca}$ is 0.032 and 0.056 , increasing $Q$ will move the detachment point from the T-junction corner to the downstream. In addition, we find the droplet detachment point gradually moves to the downstream until a stable jet is formed when we increase $\mathrm{Ca}$ and $Q$, which was also observed in both simulation [17] and 


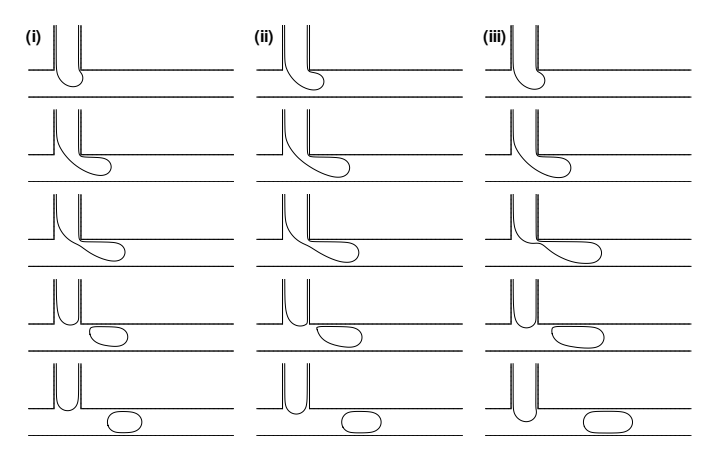

(a)

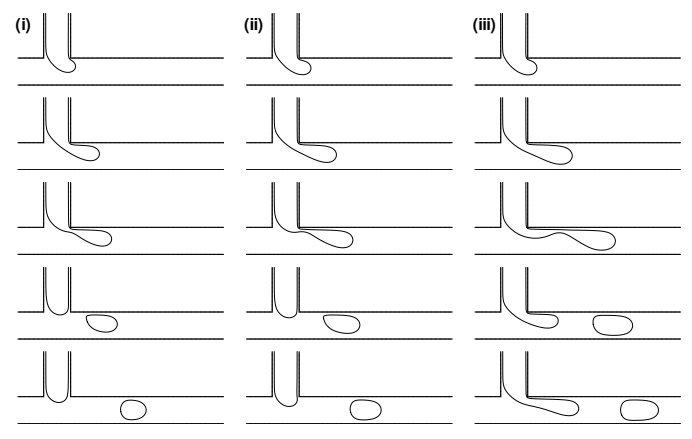

(b)

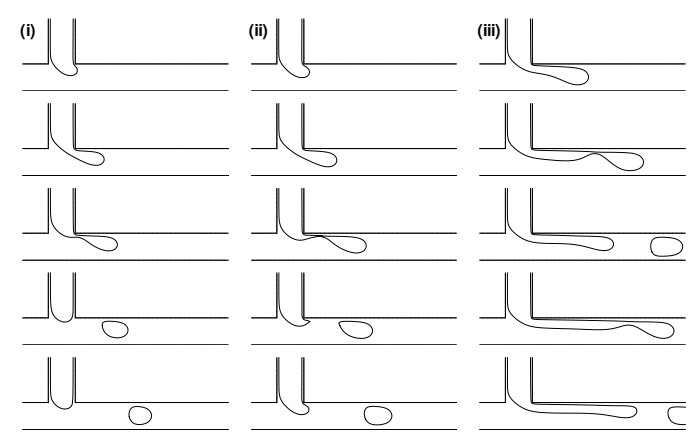

(c)

FIG. 4: The effect of capillary number and flow rate ratio in droplet formation process with a fixed viscosity ratio, i.e. $\lambda=1 / 8$ : (a) $\mathrm{Ca}=0.006$; (b) $\mathrm{Ca}=0.032$; (c) $\mathrm{Ca}=0.056$; and the flow rate ratio $Q$ is: (i) $1 / 8$; (ii) $1 / 4$; and (iii) $1 / 2$.

experiment [14].

Fig. 5 gives the predicted droplet diameter as a function of the capillary number for three different flow rate ratios. The droplet grows when the flow rate ratio increases but its diameter decreases when the capillary number increases. For all $Q$, we can clearly identify two distinguished squeezing and dripping regimes with the same critical capillary number, $\mathrm{Ca}_{c}=0.018$. In the squeezing regime, the flow rate ratio shows significant effect on the droplet size. While in the dripping regime, the effect of the flow rate ratio diminishes as Ca increases, which was also recently reported by Menech et al. [17]. The influence of the flow rate ratio was numerically investigated in [17] for both squeezing and dripping regimes, where the critical capillary number of 0.015 was found for the squeezing and dripping transition. However, the recent experimental study by Christopher et al. [14] did not observe the critical capillary number during the squeezing-to-dripping transition. In our simulation, we notice that the two regimes become difficult to distinguish as $Q$ decreases because the droplet 


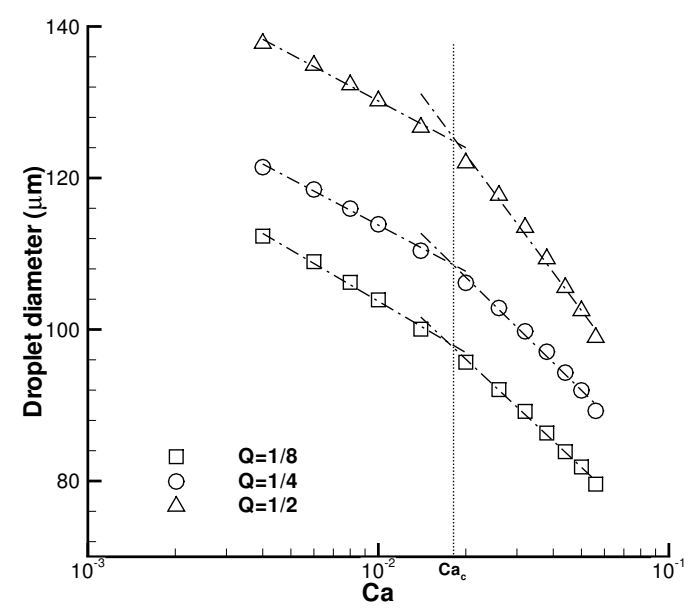

FIG. 5: The effect of capillary number and flow rate ratio on droplet diameter at a fixed viscosity ratio, $\lambda=1 / 8$.

detachment point is always close to the downstream corner of the T-junction at small $Q$. This may explain why Christopher et al. [14] did not observe the critical Ca during the squeezingto-dripping transition because they performed experiments at small viscosity ratio $\lambda=0.01$, where the droplet breakup always occurs at the downstream corner of the T-junction.

Many experimental studies were carried out in the squeezing regime so that the droplets filled the main channel and formed "plug-like" or "slug-like" shapes [5, 8, 35], where the viscous shear force may be ignored and the dominant force responsible for droplet breakup is the squeezing pressure caused by the channel obstruction. Garstecki et al. [8] argued that the detachment begins once the emerging droplet fills the main channel and the droplet continues to grow during this time due to continuous injection of the dispersed phase fluid. Assuming that the neck squeezes at a rate proportional to the average velocity of the continuous phase fluid, and the plug fills at a rate proportional to $Q_{d}$, a scaling law for the final plug length $l$ was established:

$$
l / w_{c}=1+\alpha Q
$$

where $\alpha$ is a constant of order one, whose value depends on the widths of both channels. Eq. (28) clearly shows the plug length depends only on $Q$. However, our simulation results shown in Fig. 5 suggest that the droplet size also strongly depends on Ca in the squeezing regime, which is consistent with the experimental observations [14]. Therefore, the role of 


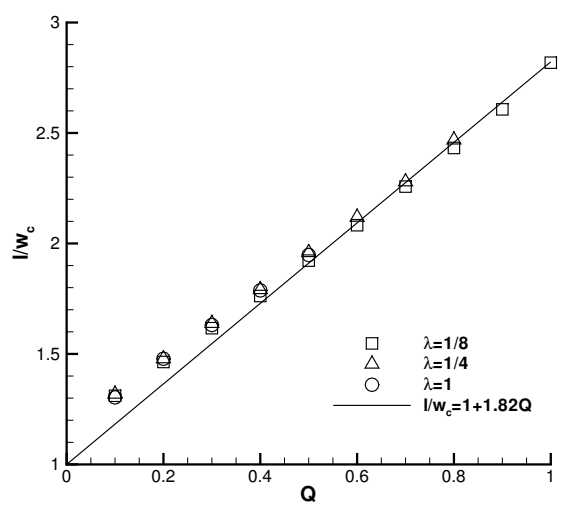

FIG. 6: For small capillary number $(\mathrm{Ca}=0.005)$ in the squeezing regime, our simulation results show that the effect of flow rate ratio on the droplet size obeys the scaling law proposed by Garstecki et al. $[8]$

capillary number needs to be reflected in the scaling law.

Although the scaling law Eq. (28) does not capture the capillary number dependency that we observe in simulations, it can predict the droplet size under various flow rate ratios when $\mathrm{Ca}$ is fixed in the squeezing regime. Fig. 6 shows a scaling formula of $l / w_{c}=1+1.82 Q$ when $\mathrm{Ca}=0.005$. In addition, Eq. (28) indicates that the droplet length is independent of the viscosity ratio $\lambda$.

\section{Influence of viscosity ratio}

Here, we investigate the role of viscosity ratio in the droplet breakup process. In the simulations, the flow rate ratio, $Q$ is fixed at $1 / 4$. The other parameters are kept the same as they are in the previous section. Fig. 7 shows a series of snapshots of droplet formation with $\lambda=1 / 8$ and 1. At the low $\mathrm{Ca}(\mathrm{Ca}=0.006)$, as shown in Fig. $7(\mathrm{a})$, the droplet fills the main channel and the detachment occurs at the downstream corner of the T-junction for both viscosity ratios. At the high capillary number $(\mathrm{Ca}=0.032,0.056)$, the large viscosity ratio will force the position of droplet detachment point to move further to the downstream, which is similar to the effects of $\mathrm{Ca}$ and $Q$ as discussed in Section B.

Fig. 8 shows that the critical capillary number $\mathrm{Ca}_{c}=0.018$, which distinguishes the squeezing and dripping regimes, is independent of the viscosity ratio. In the squeezing 


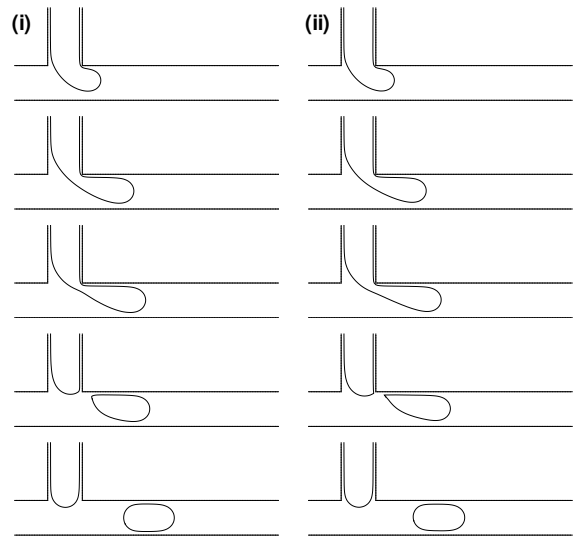

(a)

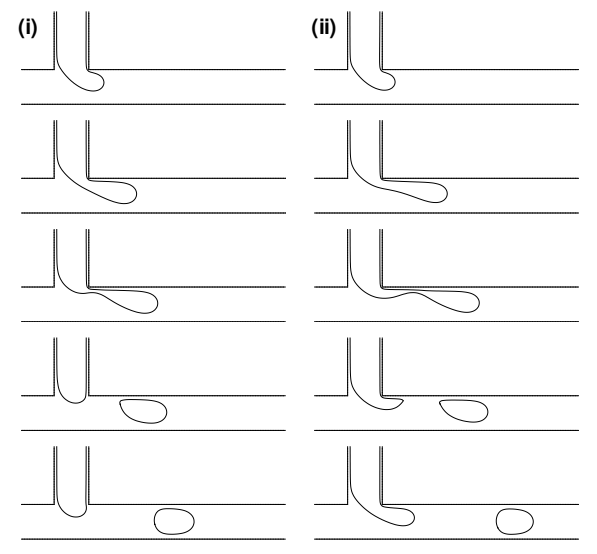

(b)

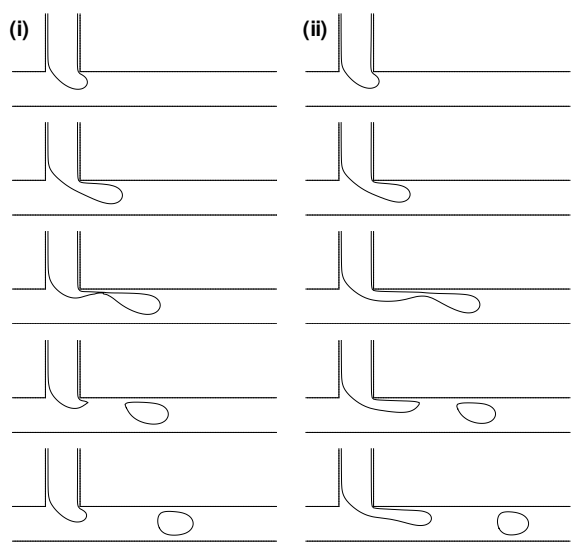

(c)

FIG. 7: The effect of viscosity ratio in the droplet formation process at a fixed flow rate ratio $Q=1 / 4$, where (a) $\mathrm{Ca}=0.006$; (b) $\mathrm{Ca}=0.032$; and (c) $\mathrm{Ca}=0.056$; and the viscosity ratio $\lambda$ is $1 / 8$ and 1 for the column (i) and (ii).

regime $\left(\mathrm{Ca} \leq \mathrm{Ca}_{c}\right)$, the predicted droplet diameter is nearly independent of the viscosity ratio, where the droplet formation is completely controlled by the capillary force and the squeezing pressure. In the dripping regime $\left(\mathrm{Ca}>\mathrm{Ca}_{c}\right)$, the influence of viscosity ratio becomes more pronounced as Ca increases, where the large viscosity ratio leads to smaller droplets. However, it also shows that the influence of viscosity ratio on the generated droplet diameter in the confined geometry is not as significant as in the unbounded flow [36], where the breakup of droplets is controlled by a competition between the viscous shear force and the capillary force in the dripping regime. This indicates that the squeezing pressure caused by the confinement of geometry of a T-junction has to be taken into account even in the dripping regime. 


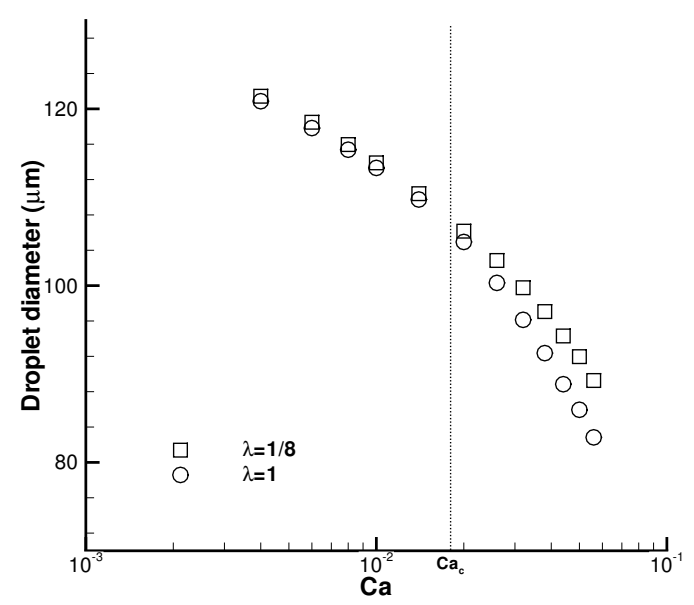

FIG. 8: The effect of capillary number and viscosity ratio on droplet diameter at a fixed flow rate ratio, i.e. $Q=1 / 4$.

\section{Influence of contact angle}

Due to high surface to volume ratio, fluid/surface interaction will significantly affect the droplet dynamics in microchannels. In order to examine the influence of wetting properties on droplet formation, we simulate the droplet generation at different contact angles i.e. $\theta_{w}=110^{\circ}, 130^{\circ}, 150^{\circ}$ and $180^{\circ}$. The other parameters are kept the same as they are in the previous section. Fig. 9 shows that the contact angle influences droplet shape, generation frequency, the distance between two neighboring droplets, and the detachment point. The droplet interfaces are prone to be normal to the channel walls at small contact angles, which is consistent with the theory of interface dynamics. In addition, our model can simulate dynamic contact angle. We find that the advancing and receding contact angles are different from the prescribed static contact angle $\theta_{w}$. This phenomenon has been observed in many experiments, and is well known as the contact angle hysteresis [37].

Fig. 10 shows that the droplet diameter decreases as the contact angle increases, but the squeezing-to-dripping transition still occurs at the the same critical capillary number i.e. $\mathrm{Ca}=0.018$ for different wetting conditions. In addition, the droplet size, which was observed to be independent of the viscosity ratio in the squeezing regime [8, 17], is only valid for more hydrophobic wetting conditions. When $\theta_{w}$ is small at $110^{\circ}$ and $130^{\circ}$, we find that the droplet size depends on the viscosity ratio. For a given contact angle $\theta_{w}$, larger $\lambda$ generally produces 


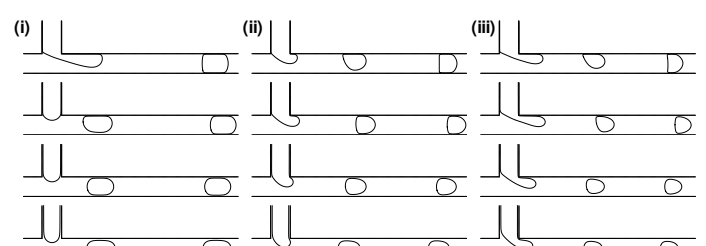

(a)

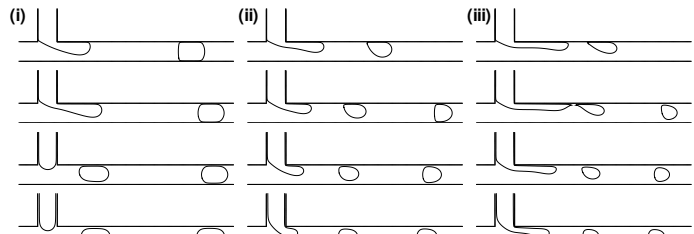

(b)

FIG. 9: The flow patterns for different contact angles: $\theta_{w}$ is same in each row $\left(110^{\circ}, 130^{\circ}, 150^{\circ}\right.$ and $180^{\circ}$ from the top row to the bottom row); column (i) $\mathrm{Ca}=0.006$, column (ii) $\mathrm{Ca}=0.032$, column (iii) $\mathrm{Ca}=0.056$; (a) $\lambda=1 / 8$ and (b) $\lambda=1$.

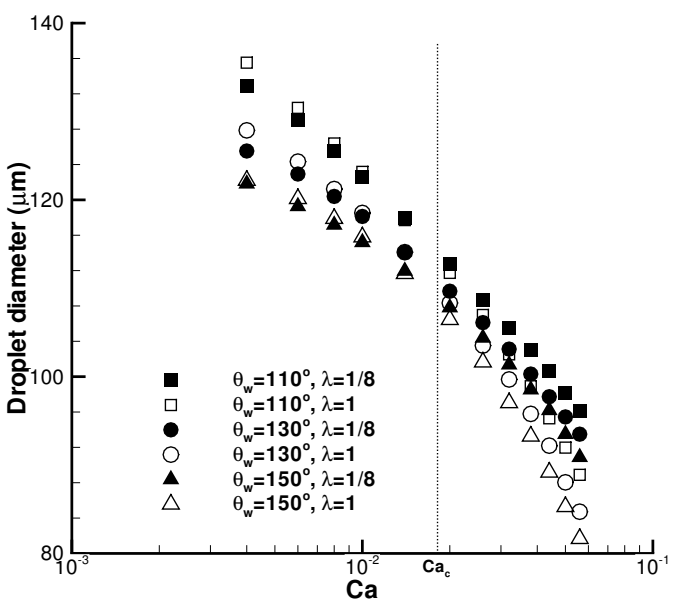

FIG. 10: The effect of contact angle, capillary number and viscosity ratio on droplet diameter.

slightly larger droplets in the squeezing regime but smaller droplets in the dripping regime. Interestingly, we find that the wetting property has more significant effect on droplet size at small $\mathrm{Ca}$, and its effect diminishes gradually when Ca increases. The reason may be that the generated droplet at a small $\mathrm{Ca}$ is usually big, which has a large contact area with the channel surface, so that the wall surface plays a more significant role in the droplet generation.

\section{CONCLUSIONS}

In this work, an improved phase field lattice Boltzmann model has been applied to study the droplet formation in a microfluidic T-junction. The capillary number, the flow rate ratio, 
the viscosity ratio and the contact angle have found to be important in droplet formation, and these parameters together control the complex droplet generation process. We have focused on droplet formation in the squeezing and dripping regimes. The squeezing-todripping transition occurs at a critical capillary number $\mathrm{Ca}_{c}=0.018$, which is independent of the flow rate ratio, the viscosity ratio and the contact angle. Unlike unbounded flow, the squeezing pressure not only dominates the droplet generation in the squeezing regime but also is important in the dripping regime. Garstecki et al. [8] confirmed that the depth of the channel plays an important role in droplet formation, so $3 D$ simulations are essential to quantitatively reveal underlying physics. In the next step, we will develop high-performance computational code using massive parallel processors to carry out 3D simulations which can capture both intermolecular and hydrodynamic effects in droplet dynamics.

[1] M. Joanicot and A. Ajdari, Sci. 309, 887 (2005).

[2] P. S. Dittrich and A. Manz, Nat. Rev. Drug Discov. 5, 210 (2006).

[3] B. T. Kelly, J. C. Baret, V. Taly, and A. D. Griffiths, Chem. Commun. 18, 1773 (2007).

[4] N. Bontoux, A. Pépin, Y. Chen, A. Ajdari, and H. A. Stone, Lab Chip 6, 930 (2006).

[5] J. D. Tice, H. Song, A. D. Lyon, and R. F. Ismagilov, Langmuir 19, 9127 (2003).

[6] T. Thorsen, R. W. Roberts, F. H. Arnold, and S. R. Quake, Phys. Rev. Lett. 86, 4163 (2001).

[7] S. van der Graaf, M. L. J. Steegmans, R. G. M. van der Sman, C. G. P. H. Schroën, and R. M. Boom, Colloids Surf. A 266, 106 (2005).

[8] P. Garstecki, M. J. Fuerstman, H. A. Stone, and G. M. Whitesides, Lab Chip 6, 437 (2006).

[9] J. Husny and J. J. Cooper-White, J. Non-Newtonian Fluid Mech. 137, 121 (2006).

[10] V. Barbier, H. Willaime, P. Tabeling, and F. Jousse, Phys. Rev. E 74, 046306 (2006).

[11] T. Nisisako, T. Torii, and T. Higuchi, Lab Chip 2, 24 (2002).

[12] T. Nisisako, T. Torii, and T. Higuchi, Chem. Eng. J. 101, 23 (2004).

[13] T. Nisisako and T. Torii, Lab Chip 8, 287 (2008).

[14] G. F. Christopher, N. N. Noharuddin, J. A. Taylor, and S. L. Anna, Phys. Rev. E 78, 036317 (2008).

[15] M. D. Menech, Phys. Rev. E 73, 031505 (2006).

[16] S. van der Graaf, T. Nisisako, C. G. P. H. Schroën, R. G. M. van der Sman, and R. M. Boom, 
Langmuir 22, 4144 (2006).

[17] M. D. Menech, P. Garstecki, F. Jousse, and H. A. Stone, J. Fluid Mech. 595, 141 (2008).

[18] L. Sang, Y. Hong, and F. Wang, Microfluid. Nanofluid. (2008).

[19] W. Shyy, R. W. Smith, H. S. Udaykumar, and M. M. Rao, Computational fluid dynamics with moving boundaries (Taylor \& Francis, Washington, DC, 1996).

[20] S. Succi, The Lattice Boltzmann Equation for Fluid Dynamics and Beyond (Oxford: Oxford University Press, 2001).

[21] X. He and L.-S. Luo, Phys. Rev. E 55, R6333 (1997).

[22] P. M. Chaikin and T. C. Lubensky, Principles of Condensed Matter Physics (Cambridge: Cambridge University Press, 2000).

[23] V. M. Kendon, M. E. Cates, I. Pagonabarraga, J. C. Desplat, and P. Bladon, J. Fluid Mech. 440, 147 (2001).

[24] J. S. Rowlinson and B. Widom, Molecular Theory of Capillarity (London: Clarendon Press, 1989).

[25] Y. H. Qian, D. D'Humières, and P. Lallemand, Europhys. Lett. 17, 479 (1992).

[26] R. G. M. van der Sman, Phys. Rev. E 74, 026705 (2006).

[27] A. J. C. Ladd, J. Fluid Mech. 271, 285 (1994).

[28] Q. Zou and X. He, Phys. Fluids 9, 1591 (1997).

[29] D. Iwahara, H. Shinto, M. Miyahara, and K. Higashitani, Langmuir 19, 9086 (2003).

[30] C. M. Pooley and K. Furtado, Phys. Rev. E 77, 046702 (2008).

[31] J. W. Cahn, J. Chem. Phys. 66, 3667 (1977).

[32] R. Dreyfus, P. Tabeling, and H. Willaime, Phys. Rev. Lett. 90, 144505 (2003).

[33] J. H. Xu, G. S. Luo, S. W. Li, and G. G. Chen, Lab Chip 6, 131 (2005).

[34] J. H. Xu, G. S. Luo, G. G. Chen, and J. D. Wang, J. Membr. Sci. 266, 121 (2005).

[35] B. Zheng, J. D. Tice, and R. F. Ismagilov, Anal. Chem. 76, 4977 (2004).

[36] P. B. Umbanhowar, V. Prasad, and D. A. Weitz, Langmuir 16, 347 (2000).

[37] H. J. Butt, K. Graf, and M. Kappl, Physics and Chemistry of Interfaces (Wiley-VCH, 2003). 


\section{CAPTIONS}

Figure 1. Schematic illustration of the droplet generation in a microfluidic T-junction, where $w_{c}, w_{d}$ are the widths of the main and lateral channels, and $Q_{c}$ and $Q_{d}$ are the inlet volumetric flow rates of the continuous and dispersed phases.

Figure 2. The snapshots of droplet formation: (a) $\mathrm{Ca}=0.00056, Q_{d}=0.004$; and (b) $\mathrm{Ca}=0.059, Q_{d}=0.008$.

Figure 3. The effect of capillary number on the droplet diameter at the dispersed phase flow rates $Q_{d}$ of $4 \times 10^{-3}, 6 \times 10^{-3}$ and $8 \times 10^{-3}$ respectively. The lines represent the power fitting of the simulation results

Figure 4. The effect of capillary number and flow rate ratio in droplet formation process with a fixed viscosity ratio, i.e. $\lambda=1 / 8$ : (a) $\mathrm{Ca}=0.006$; (b) $\mathrm{Ca}=0.032$; (c) $\mathrm{Ca}=0.056$; and the flow rate ratio $Q$ is: (i) $1 / 8$; (ii) $1 / 4$; and (iii) $1 / 2$.

Figure 5. The effect of capillary number and flow rate ratio on droplet diameter at a fixed viscosity ratio, $\lambda=1 / 8$.

Figure 6. For small capillary number $(\mathrm{Ca}=0.005)$ in the squeezing regime, our simulation results show that the effect of flow rate ratio on the droplet size obeys the scaling law proposed by Garstecki et al. [8].

Figure 7. The effect of viscosity ratio in the droplet formation process at a fixed flow rate ratio $Q=1 / 4$, where (a) $\mathrm{Ca}=0.006$; (b) $\mathrm{Ca}=0.032$; and (c) $\mathrm{Ca}=0.056$; and the viscosity ratio $\lambda$ is $1 / 8$ and 1 for the column (i) and (ii).

Figure 8. The effect of capillary number and viscosity ratio on droplet diameter at a fixed flow rate ratio, i.e. $Q=1 / 4$.

Figure 9. The flow patterns for different contact angles: $\theta_{w}$ is same in each row $\left(110^{\circ}, 130^{\circ}, 150^{\circ}\right.$ and $180^{\circ}$ from the top row to the bottom row); column (i) Ca=0.006, column (ii) $\mathrm{Ca}=0.032$, column (iii) $\mathrm{Ca}=0.056$; (a) $\lambda=1 / 8$ and (b) $\lambda=1$.

Figure 10. The effect of contact angle, capillary number and viscosity ratio on droplet diameter. 